\title{
Comparison and validation of the value of preoperative inflammation marker-based prognostic scores in resectable pancreatic ductal adenocarcinoma
}

This article was published in the following Dove Press journal: Cancer Management and Research

\author{
Sisi Ye \\ Li Bai \\ Department of Medical Oncology, \\ Chinese PLA General Hospital, \\ Beijing 100853, China
}

Correspondence: Li Bai Department of Medical Oncology, Chinese PLA General Hospital, 28 Fuxing Road, Beijing 100853, China

Tel +0II 86 I77 I0I7 204I

Fax +0II 861066937876

Email baili_30I@I63.com
Purpose: We aimed to compare and validate the prognostic value of inflammation-based prognostic scores for patients with resectable pancreatic ductal adenocarcinoma (PDAC) and to establish a novel, effective nomogram incorporating inflammation-based prognostic scores to predict disease-free survival (DFS) in these patients.

Patients and methods: Clinicopathological characteristics and follow-up data were retrieved from our prospective database. A total of 420 patients with PDAC who underwent radical resection were evaluated between January 2007 and December 2016. Blood samples were obtained within 7 days prior to surgical intervention. Time-dependent receiver operating characteristic curves were generated to determine optimal cutoff values for independent prognostic factors. Nomograms for DFS were established based on multivariate Cox proportional hazard models, and the results were internally validated using bootstrap resampling.

Results: Neutrophil-lymphocyte ratio (NLR) $>2.13$ and platelet-lymphocyte ratio (PLR) $>140$ were independent prognostic factors for DFS and overall survival. Stratified analyses indicated that these associations were not present among patients with stage III disease. Independent predictors of DFS, including Eastern Cooperative Oncology Group performance status score, tumor stage, differentiation, adjuvant treatment, NLR, and PLR, were incorporated into two nomograms for prediction of DFS in a cohort of PDAC patients after curative surgery. The area under receiver operating characteristic curve of the nomogram including NLR and PLR (0.804) was higher than that without NLR and PLR (0.711). The bootstrap-corrected AUC for the model including NLR and PLR was 0.803, while that for the model without NLR and PLR was 0.711. Conclusion: Our study demonstrates that high NLR and high PLR are important clinical predictors of poor survival in patients with resectable PDAC. Additionally, a nomogram including NLR and PLR can objectively and reliably predict DFS among patients with PDAC following curative surgery.

Keywords: neutrophil-lymphocyte ratio, NLR, platelet-lymphocyte ratio, PLR, nomogram, disease-free survival

\section{Introduction}

Pancreatic ductal adenocarcinoma (PDAC) ranks third among the causes of cancerrelated death, with approximately 338,000 new cases annually worldwide. ${ }^{1}$ Prognosis remains poor, and the 5-year cumulative survival rates for PDAC are estimated at less than $10 \%{ }^{2}$ Surgical resection offers the only chance of cure; however, over $80 \%$ of patients experience local or distant recurrence after radical surgery. ${ }^{3}$ Current predictions 
of tumor recurrence depend primarily on the histopathological characteristics of resected tumor specimens, including tumor size, histological grade, the depth of infiltration, and lymph node involvement; however, patients with equivalent pathological status often exhibit highly heterogeneous survival rates, suggesting that conventional characteristics are insufficient for optimal prediction of an individual's prognosis. ${ }^{4-6}$ Hence, the identification of novel prognostic factors to enable a better risk stratification for follow-up schedules and individualized treatment of PDAC, has become critical.

Inflammation is a recognized hallmark of various malignant solid tumors, including PDAC. ${ }^{7}$ Chronic inflammation contributes substantially to the development and progression of malignancies, through its influence on tumor angiogenesis, invasion, and metastasis. ${ }^{8}$ Conversely, malignancies can also trigger regional inflammatory responses and the release of pro-inflammatory cytokines, leading to the formation of an inflammatory tumor microenvironment which favors tumor progression. ${ }^{8,9}$ Several prognostic factors based on analysis of systemic inflammation scores, including neutrophil-lymphocyte ratio (NLR), platelet-lymphocyte ratio (PLR), lymphocyte-monocyte ratio (LMR), and prognostic nutritional index (PNI), have been proposed to estimate the magnitude of systemic inflammation in patients with cancer. ${ }^{10,11}$ These proposed biomarkers are potentially useful prognostic indicators for stratification of patients with good and poor prognosis. ${ }^{10,12,13}$ The results of published studies that have examined the relationship between inflammationbased prognostic indices and resectable PDAC have been somewhat inconsistent to date. ${ }^{12,14,15}$ Possible explanations for these inconsistencies may include the relatively small numbers included in these studies $(n<100)$, differences in inclusion criteria and clinical endpoints, unclear selection of cutoff values, and concomitant evaluation of resectable and unresectable disease; these limitations would affect the reproducibility and reliability of these markers. Furthermore, inflammation-based prognostic indices have yet to be appropriately validated for clinical decision-making. Therefore, the aim of this study was to compare the prognostic values of NLR, PLR, LMR, and PNI and to develop a nomogram incorporating inflammation-based prognostic indices to predict the disease-free survival (DFS) in a large cohort of 420 patients with PDAC who underwent curative resection.

\section{Materials and methods}

\section{Study population}

We conducted a retrospective study of patients with PDAC who underwent potentially radical resection from January
2007 to December 2016 at the Chinese PLA General Hospital, Beijing, China. Patients were excluded from the study if they met the following criteria: 1) without pathologically confirmed PDAC; 2) positive surgical margins; 3) presence of distant metastasis; 4) preoperative chemotherapy or radiotherapy for PDAC; 5) other malignancies diagnosed within 5 years; 6) clinical evidence of acute infection or other inflammatory conditions; 7) previous hematological or autoimmune disease; and 8) no complete record of preoperative hematological variables and follow-up data. Following these exclusions, a total of 420 patients with PDAC were eligible for the present study. Approval for the study was granted by the ethics committee of the Chinese PLA General Hospital, and the requirement to obtain written informed consent from patients was waived due to the retrospective nature of the investigation. To protect patient privacy, all personal information was appropriately anonymized and de-identified prior to analysis.

\section{Data collection and clinical definition}

Information regarding the clinicopathological characteristics, sociodemographic data, and lifestyle factors were retrieved from medical records. Laboratory data, including routine blood examination, albumin concentration, and levels of the tumor markers, CA19-9, CA125, and CEA, were obtained within 7 days prior to surgical intervention. Pathological data included tumor size, histological type, grade, the presence of micro-invasion (of nerves, lymphatics, and vessels), number of cancer positive lymph nodes, and microscopic status of all evaluated resection margins. Pathological staging was performed according to the tumor-node-metastasis (TNM) classification system; as the TNM classification system for PDAC changed during the study period, tumors were uniformly staged according to the eighth edition of this system. Alcohol intake was classified as either currently drinking almost/more than once a week or not. Smoking habit was defined as either smoking more than once a day or not. Body mass index (BMI) was calculated as weight divided by height squared (in $\mathrm{kg} \mathrm{m}^{2}$ ). NLR was defined as the absolute neutrophil count divided by absolute lymphocyte count. PLR was calculated as the absolute platelet count divided by the absolute lymphocyte count. LMR was determined as the absolute lymphocyte count divided by absolute monocyte count. PNI was calculated as $10 \times$ albumin $(\mathrm{g} / \mathrm{dL})+0.005 \times$ total lymphocyte count (per $\mathrm{mm}^{3}$ ).

Follow-up data were collected prospectively, and all patients were followed-up every 3 months during the first and second postoperative year, every 6 months in the third to fifth 
years, and annually thereafter. If recurrence was confirmed, the follow-up interval was 3 months. The closing date for follow-up was February 30, 2018. The primary endpoint of the study was DFS. The secondary endpoint was overall survival (OS). DFS was defined as the time from surgical resection to local recurrence or distant metastasis. OS was defined as the interval between the date of a definitive diagnosis and cancer-related death. Patients were censored at the date of last follow-up.

\section{Statistical analysis}

Continuous variables are expressed as median (25th, 75th percentile) and categorical variables as frequency and percentage. Differences in the distribution of categorical variables were evaluated using the chi-squared test. Time-dependent receiver operating characteristic (ROC) curves were generated to determine the optimal cutoff values of independent prognostic factors. Kaplan-Meier analyses with log-rank tests were used to compare survival outcomes between patients with systemic inflammatory marker values above vs below optimal cutoff points. Cox proportional hazard models were used to assess the relationships between systemic inflammatory markers and survival outcomes. Unadjusted and multivariate adjusted models were applied, and HR and 95\% confidence intervals (CIs) calculated. Nomograms for predicting 1-year DFS were established based on the results of multivariate Cox proportional hazard regression models. A backward model selection technique was used for the final model selection, and predictive accuracy was evaluated by calculation of the area under the ROC curve. The performance of the models was internally validated using bootstrapping (500 samples). Statistical significance was defined as a twotailed $P$-value $<0.05$.

All statistical analyses were undertaken using the statistical software packages, R (http://www.R-project.org, The R Foundation) and EmpowerStats (http://www.empowerstats. com, X\&Y Solutions, Inc., Boston, MA, USA).

\section{Results}

Table 1 summarizes the detailed clinical characteristics of the 420 eligible patients. The median follow-up time was 38 months (range 1-75 months). Of the 420 resectable PDAC patients, 382 (91\%) developed tumor recurrence and 346 (82.4\%) died within the follow-up period. The median DFS and OS were 9.2 and 14.4 months, respectively.

The AUCs of the time-dependent ROC curve analyses are presented in Table 2 and ROC curves are shown in Figure 1. The AUCs for NLR and PLR were 0.743 and 0.715 ,
Table I Baseline characteristics of patients

\begin{tabular}{|c|c|}
\hline Characteristic & Total patients $(n=420)$ \\
\hline \multicolumn{2}{|l|}{ Age (years), n (\%) } \\
\hline$\leq 50$ & $64(15.2)$ \\
\hline $5 I-70$ & $308(73.3)$ \\
\hline$>70$ & $48(14.4)$ \\
\hline \multicolumn{2}{|l|}{ Sex, n (\%) } \\
\hline Female & $156(37.1)$ \\
\hline Male & $264(62.9)$ \\
\hline \multicolumn{2}{|l|}{ BMI (kg/m²), n (\%) } \\
\hline$<18.5$ & 47 (II.2) \\
\hline $18.5-24$ & $318(75.7)$ \\
\hline$\geq 24$ & $55(\mid 3 . I)$ \\
\hline \multicolumn{2}{|l|}{ Smoking, n (\%) } \\
\hline Yes & $74(17.6)$ \\
\hline No & $346(82.4)$ \\
\hline \multicolumn{2}{|l|}{ Alcohol consumption, n (\%) } \\
\hline Yes & $126(30)$ \\
\hline No & $294(70)$ \\
\hline \multicolumn{2}{|l|}{ ECOG PS, n (\%) } \\
\hline $0-1$ & $356(84.8)$ \\
\hline$\geq 2$ & $64(15.2)$ \\
\hline \multicolumn{2}{|l|}{ Tumor location, n (\%) } \\
\hline Head & $286(68.1)$ \\
\hline Body and/or tail & $134(31.9)$ \\
\hline \multicolumn{2}{|l|}{ Operation } \\
\hline Pancreaticoduodenectomy & $286(68.1)$ \\
\hline Distal pancreatectomy & $132(31.4)$ \\
\hline Total pancreatectomy & $2(0.05)$ \\
\hline \multicolumn{2}{|l|}{ Maximum diameter $(\mathrm{cm}), \mathrm{n}(\%)$} \\
\hline$<2$ & $31(7.4)$ \\
\hline $2-4$ & $257(6 \mid .2)$ \\
\hline$>4$ & $132(3 \mid .4)$ \\
\hline \multicolumn{2}{|l|}{ Differentiation, n (\%) } \\
\hline Well & $225(53.6)$ \\
\hline Poor & $195(46.4)$ \\
\hline \multicolumn{2}{|l|}{ T stage, n (\%) } \\
\hline TI & $45(10.7)$ \\
\hline $\mathrm{T} 2$ & $232(55.3)$ \\
\hline T3 & $132(3 \mid .4)$ \\
\hline $\mathrm{T} 4$ & II (2.6) \\
\hline \multicolumn{2}{|l|}{$\mathrm{N}$ stage, $\mathrm{n}(\%)$} \\
\hline No & $28 I(66.9)$ \\
\hline $\mathrm{NI}$ & $103(24.5)$ \\
\hline N2 & $36(8.6)$ \\
\hline \multicolumn{2}{|l|}{ pTNM stage, n (\%) } \\
\hline I & $186(44.3)$ \\
\hline II & $188(44.8)$ \\
\hline III & $46(10.9)$ \\
\hline \multicolumn{2}{|l|}{ Tumor extension, n (\%) } \\
\hline Choledochal duct & $150(35.7)$ \\
\hline Duodenum & $146(34.8)$ \\
\hline Peripheral tissue & $158(37.6)$ \\
\hline Nerve & $124(29.5)$ \\
\hline Vessel & $28(6.7)$ \\
\hline \multicolumn{2}{|l|}{ Tumor thrombus, $\mathrm{n}(\%)$} \\
\hline Yes & $50(11.9)$ \\
\hline No & $370(11.9)$ \\
\hline
\end{tabular}

(Continued) 
Table I (Continued)

\begin{tabular}{ll}
\hline Characteristic & Total patients $(\mathbf{n = 4 2 0})$ \\
\hline Adjuvant treatment & \\
Yes & $117(27.9)$ \\
No & $303(72.1)$ \\
NLR, median (IQR) & $2.12(1.53-3.1 \mathrm{I})$ \\
PLR, median (IQR) & $127.94(92.10-175.56)$ \\
LMR, median (IQR) & $4.53(3.20-5.70)$ \\
PNI, median (IQR) & $48.39(45.45-51.84)$ \\
CAI9-9 (U/mL), n (\%) & \\
$\leq 37$ & $89(21.2)$ \\
$>37$ & $331(78.8)$ \\
CAI25 (U/mL) & \\
$\leq 35$ & $341(81.2)$ \\
$>35$ & $79(18.8)$ \\
CEA $(\mathrm{ng} / \mathrm{mL})$ & \\
$\leq 5$ & $284(67.6)$ \\
$>5$ & $136(32.4)$ \\
\hline
\end{tabular}

Note: Continuous data are presented as median (interquartile range) and categorical data are shown as frequency (\%).

Abbreviations: CAI9-9, carbohydrate antigen 19-9; CAI25, carbohydrate antigen 125; CEA, carcinoembryonic antigen; BMI, body mass index; ECOG PS, Eastern Cooperative Oncology Group performance status; IQR, interquartile range; LMR, lymphocyte-monocyte ratio; NLR, neutrophil-lymphocyte ratio; PLR, plateletlymphocyte ratio; $\mathrm{PNI}$, prognostic nutritional index.

respectively. The optimal cutoff value for NLR was 2.13, with a sensitivity of $61.7 \%$ and a specificity of $74.1 \%$ for 1 -year DFS. For PLR, the optimal cutoff value was 140 with a sensitivity of $54.1 \%$ and a specificity of $80.6 \%$. LMR and PNI were not suitable for inclusion in subsequent analyses since their AUC values were $<0.5$.

Patients were divided into low or high groups based on NLR and PLR cutoff values. Comparisons of the baseline characteristics of patients stratified by NLR and PLR are presented in Table 3. Compared to low NLR $(<2.13)$, high NLR (>2.13) was significantly associated with unfavorable tumor characteristics including higher $\mathrm{T}$ stage, more positive lymph nodes, and advanced stage. Similar significant differences were also observed when the two groups were stratified by PLR.

Kaplan-Meier analyses demonstrated that high preoperative NLR and PLR were associated with worse DFS and OS. The median DFS and OS of patients with an NLR of $\leq 2.13$ was 12.4 and 23.1 months, respectively, whereas for patients with an NLR of $>2.13$ the equivalent values were 6.0 and 10.7 months, respectively (both $P<0.01$; Figure $2 \mathrm{~A}$ and C). Patients with PLR $\leq 140$ had superior DFS (12.1 vs 5.9 months, $P<0.001)$ and OS (23.5 vs 10.6 months, $P<0.001)$ compared to those with PLR $>140$ (Figure $2 \mathrm{~B}$ and D).

The results of univariate and multivariable Cox regression analyses are presented in Table 4. Both high NLR and high
PLR were identified as independently associated with inferior DFS and OS in unadjusted analysis. The adjusted HR (95\% CI), $P$-value of worse DFS and OS for patients with high NLR were 1.59 (1.27-2.00), <0.001 and $1.70(1.33-2.16),<0.001$, respectively. Patients with high PLR had inferior DFS (HR [95\% CI]: 1.93 [1.53-2.45], $P<0.001)$ and OS (HR [95\% CI]: 2.23 [1.74-2.85], $P<0.001)$ compared to their counterparts with low PLR.

Given the close relationship between survival outcomes and tumor stage, we also performed an analysis with data stratified by tumor stage subgroups (Table 5). Both high NLR and high PLR remained independent risk factors for inferior DFS and OS in patients with stage I and II tumors; however, for patients with stage III disease, these associations were lost after adjustment for confounding factors.

To predict the 1-year DFS of patients with resectable PDAC, nomograms (Figure 3A and B) were established using a multivariate Cox regression model incorporating all significant independent predictors of DFS. Eastern Cooperative Oncology Group performance status (ECOG PS) score, stage, differentiation, adjuvant treatment, NLR, and PLR were incorporated into the DFS nomograms. The AUCs of the nomograms, either including or excluding NLR and PLR, were 0.804 and 0.711 , respectively (Figure $3 \mathrm{C}$ and $\mathrm{D}$ ). The respective $\mathrm{C}$-indices of the nomograms including or excluding NLR and PLR were 0.750 and 0.679 . The discriminative performance of the nomogram (bootstrap-corrected AUC) was 0.803 for the model including NLR and PLR, and 0.711 for that excluding NLR and PLR.

\section{Discussion}

In the present study, we demonstrated that NLR $>2.13$ and PLR > 140 can be reliable predictive markers for shorter DFS and OS in a large and homogenous population of patients with resectable PDAC. After adjustment for a wide range of potential confounding factors, NLR $>2.13$ and PLR $>140$ remained independent unfavorable prognostic factors with clinically relevant HR values; however, these associations were not consistent among the patient subgroup with stage III tumors. Additionally, we presented a nomogram including NLR and PLR that predicts the 1-year DFS with good discriminative performance. To the best of our knowledge, this is the first nomogram for patients with resected PDAC incorporating inflammation-based prognostic indices to effectively predict DFS.

It is well-established that cancer progression is determined not only by oncogenes or tumor suppressor genes within the tumor itself but also by host-related factors such as 
Table 2 ROC curve analyses of the NLR, PLR, LMR, and PNI for I-year DFS

\begin{tabular}{llllll}
\hline Variable & Cutoff & AUC & Sensitivity & Specificity & Harrell's C index \\
\hline NLR & 2.13 & 0.743 & 0.617 & 0.741 & 0.699 \\
PLR & 140 & 0.715 & 0.541 & 0.806 & 0.668 \\
LMR & - & 0.297 & 1 & 0 & 0.652 \\
PNI & 64.96 & 0.397 & 0.006 & 0.998 & 0.599 \\
\hline
\end{tabular}

Abbreviations: AUC, area under the curve; DFS, disease-free-survival; LMR, lymphocyte-monocyte ratio; NLR, neutrophil-lymphocyte ratio; PLR, platelet-lymphocyte ratio; $\mathrm{PNI}$, prognostic nutritional index; ROC, receiver operating characteristic.

A

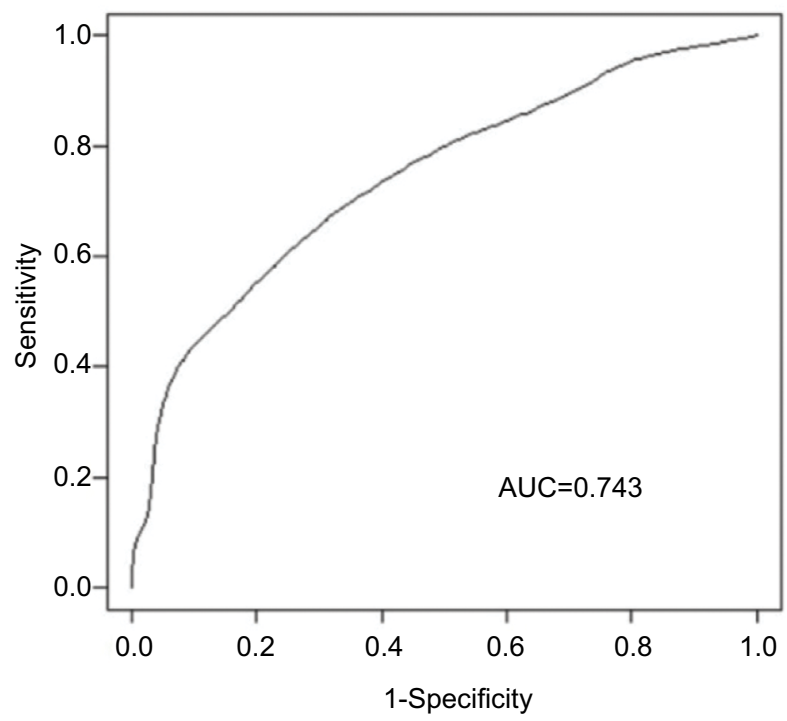

C

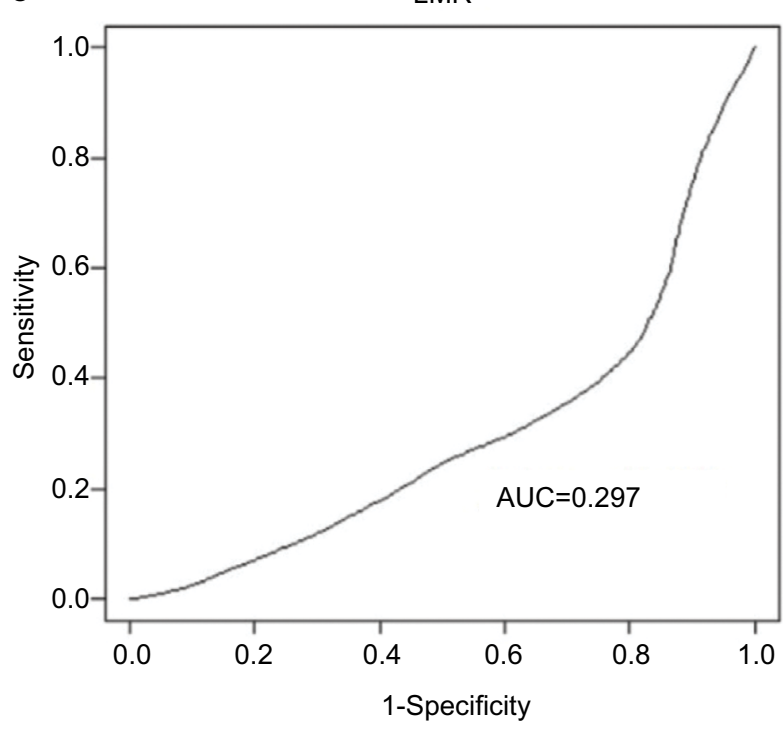

B

PLR

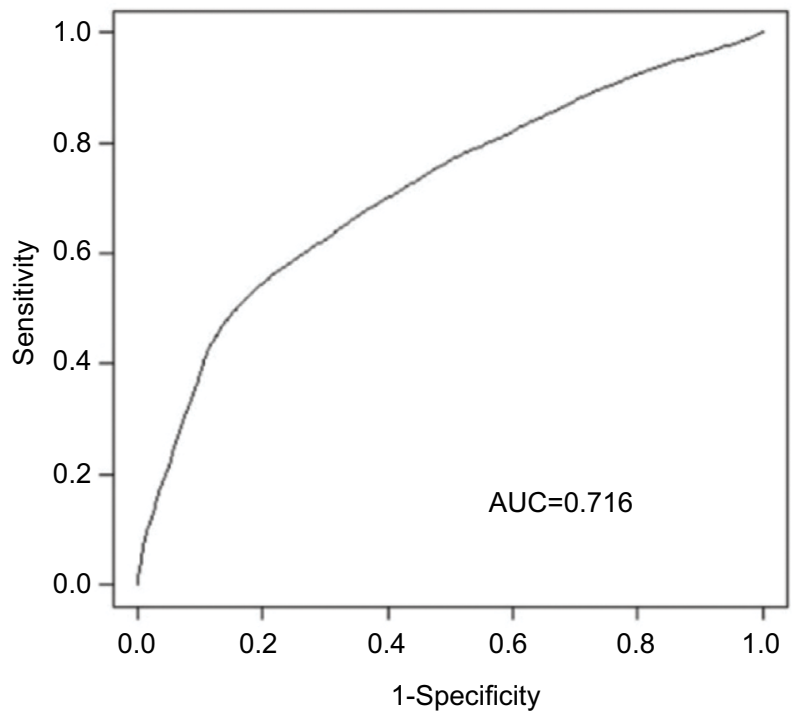

D

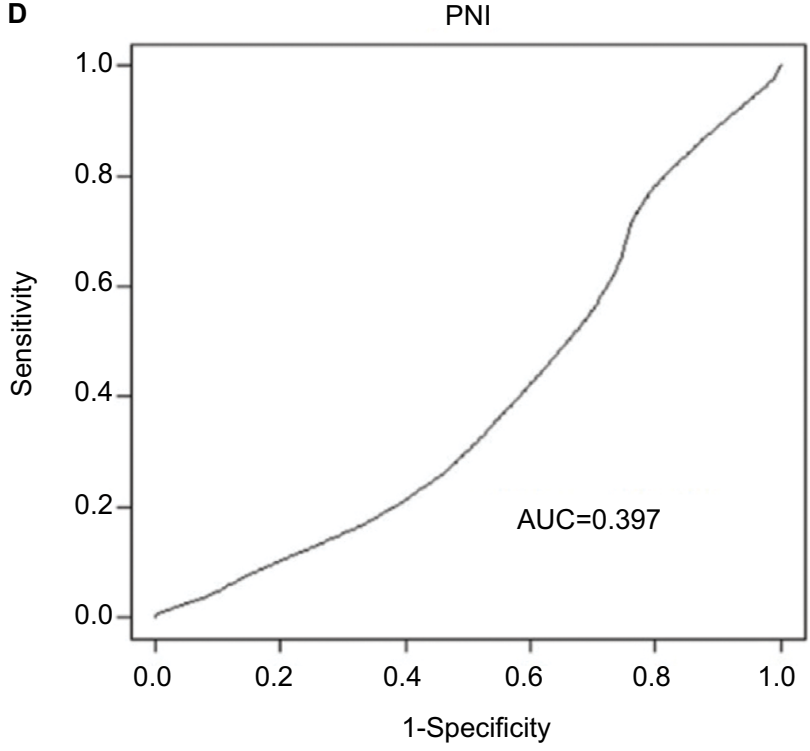

Figure I ROC curves of NLR, PLR, LMR, and PNI for I-year DFS.

Note: (A) NLR, (B) PLR, (C) LMR, and (D) PNI.

Abbreviations: AUC, area under the curve; DFS, disease-free survival; LMR, lymphocyte-monocyte ratio; NLR, neutrophil-lymphocyte ratio; PLR, platelet-lymphocyte ratio; PNI, prognostic nutritional index; ROC, receiver operating characteristic.

age, sex, performance status, and inflammatory responses. ${ }^{16}$ The relationship between systemic inflammation and cancer progression has been subject to increasing attention in investigation of various gastrointestinal malignancies, including PDAC. ${ }^{17,18}$ Cancer-related inflammation involves crosstalk of inflammatory cells and mediators, such as cytokines 
Table 3 Baseline characteristics stratified by NLR and PLR

\begin{tabular}{|c|c|c|c|c|c|c|}
\hline \multirow[t]{2}{*}{ Characteristic } & \multicolumn{3}{|l|}{ NLR } & \multicolumn{3}{|l|}{ PLR } \\
\hline & $\leq 2.13(n=215)$ & $>2.13(n=205)$ & $P$-value & $\leq 140(n=245)$ & $>140(n=175)$ & $P$-value \\
\hline \multicolumn{7}{|l|}{ Age (years), n (\%) } \\
\hline$\leq 50$ & 25 (II.6) & $39(19)$ & 0.043 & $4 I(16.7)$ & $23(13.1)$ & 0.003 \\
\hline $51-70$ & $160(74.4)$ & I 48 (72.2) & & $166(67.8)$ & I42 (8I. $)$ & \\
\hline$>70$ & $30(14)$ & $18(8.8)$ & & $38(15.5)$ & $10(5.8)$ & \\
\hline \multicolumn{7}{|l|}{ Sex, n (\%) } \\
\hline Female & $83(38.6)$ & $73(35.6)$ & 0.525 & $86(35.1)$ & $70(40)$ & 0.306 \\
\hline Male & $132(6 \mid .4)$ & $132(64.4)$ & & $159(64.9)$ & $105(60)$ & \\
\hline \multicolumn{7}{|l|}{ BMI $\left(\mathrm{kg} / \mathrm{m}^{2}\right), \mathrm{n}(\%)$} \\
\hline$<18.5$ & $32(14.9)$ & $15(7.3)$ & $<0.001$ & $35(14.3)$ & $12(6.9)$ & $<0.001$ \\
\hline $18.5-24$ & $144(67)$ & $174(84.9)$ & & $167(68.2)$ & I5I (86.3) & \\
\hline$\geq 24$ & $39(18.1)$ & $16(7.8)$ & & $43(17.6)$ & $12(6.8)$ & \\
\hline \multicolumn{7}{|l|}{ Smoking, n (\%) } \\
\hline Yes & $37(17.2)$ & $37(18)$ & 0.821 & $46(18.8)$ & $28(16)$ & 0.462 \\
\hline No & $178(82.8)$ & $168(82)$ & & $199(81.2)$ & $147(84)$ & \\
\hline \multicolumn{7}{|l|}{ Alcohol consumption, $\mathrm{n}(\%)$} \\
\hline Yes & $62(28.8)$ & $64(3 \mid .2)$ & 0.594 & 79 (32.2) & $47(26.9)$ & 0.235 \\
\hline No & I53 (7I.2) & I4I (68.8) & & $166(67.8)$ & $128(73.1)$ & \\
\hline \multicolumn{7}{|l|}{ ECOG PS, n (\%) } \\
\hline $0-1$ & $202(94)$ & $154(75.1)$ & $<0.001$ & $223(91)$ & $133(76)$ & $<0.001$ \\
\hline$\geq 2$ & $13(6)$ & $51(24.9)$ & & $22(9)$ & $42(24)$ & \\
\hline \multicolumn{7}{|l|}{ Tumor location, n (\%) } \\
\hline Head & $147(68.4)$ & $139(67.8)$ & 0.901 & $|5|(6 \mid .6)$ & I $35(77.1)$ & $<0.001$ \\
\hline Body and/or tail & $68(31.6)$ & $66(32.2)$ & & $94(38.4)$ & $40(22.9)$ & \\
\hline \multicolumn{7}{|l|}{ Operations } \\
\hline Pancreaticoduodenectomy & 147 (68.4) & $139(67.8)$ & 0.876 & $|5|(6 \mid .6)$ & I $35(77.1)$ & $<0.001$ \\
\hline Distal pancreatectomy & $66(30.7)$ & $66(32.2)$ & & $93(38)$ & $39(22.3)$ & \\
\hline Total pancreatectomy & $2(0.9)$ & $0(0)$ & & I (0.4) & I (0.6) & \\
\hline \multicolumn{7}{|l|}{ Maximum diameter (cm), n (\%) } \\
\hline$<2$ & I8 (8.4) & $13(6.3)$ & 0.707 & $20(8.2)$ & II (6.3) & $0.27 \mathrm{I}$ \\
\hline $2-4$ & $129(60)$ & $128(62.4)$ & & $142(58)$ & $115(65.7)$ & \\
\hline$>4$ & $68(31.6)$ & $64(31.3)$ & & $83(33.8)$ & $49(28)$ & \\
\hline \multicolumn{7}{|l|}{ Differentiation, $\mathrm{n}(\%)$} \\
\hline Well & $120(55.8)$ & $105(5 \mid .2)$ & 0.345 & $129(52.7)$ & $96(54.9)$ & 0.655 \\
\hline Poor & $95(44.2)$ & $100(48.8)$ & & $116(47.3)$ & $79(45.1)$ & \\
\hline \multicolumn{7}{|l|}{ T stage, n (\%) } \\
\hline TI & $28(13)$ & $17(8.3)$ & 0.003 & $31(12.7)$ & $14(8)$ & $<0.001$ \\
\hline $\mathrm{T} 2$ & $119(55.3)$ & $113(55.1)$ & & $|3|(53.5)$ & 101 (57.7) & \\
\hline T3 & $68(31.7)$ & $64(3 \mid .2)$ & & $83(33.9)$ & $49(28)$ & \\
\hline $\mathrm{T} 4$ & $0(0)$ & II (5.4) & & $0(0)$ & II (6.3) & \\
\hline \multicolumn{7}{|l|}{ N stage, n (\%) } \\
\hline No & I54 (7I.6) & $127(62)$ & 0.019 & $173(70.6)$ & $108(6 \mid .7)$ & 0.03 \\
\hline $\mathrm{NI}$ & $50(23.3)$ & $53(25.9)$ & & $58(23.7)$ & $45(25.7)$ & \\
\hline N2 & II (5.I) & $25(12.1)$ & & $14(5.7)$ & $22(12.6)$ & \\
\hline \multicolumn{7}{|l|}{ pTNM stage, n (\%) } \\
\hline I & $105(48.8)$ & $81(39.5)$ & $<0.001$ & II 4 (46.5) & $72(4 I . I)$ & $<0.001$ \\
\hline ॥ & $99(46)$ & 89 (43.4) & & $117(47.8)$ & $71(40.6)$ & \\
\hline III & II (5.I) & $35(17.1)$ & & $14(5.7)$ & $32(18.3)$ & \\
\hline \multicolumn{7}{|l|}{ Tumor extension, $\mathrm{n}(\%)$} \\
\hline Choledochal duct & $85(39.5)$ & $65(31.7)$ & 0.094 & $96(39.2)$ & $54(30.9)$ & 0.079 \\
\hline Duodenum & $80(37.2)$ & $66(32.2)$ & $0.28 I$ & $88(35.9)$ & $58(33.1)$ & 0.556 \\
\hline Peripheral tissue & $72(33.5)$ & $86(42)$ & 0.074 & $86(35.1)$ & $72(4 I . I)$ & 0.208 \\
\hline Nerve & $68(31.6)$ & $56(27.3)$ & 0.333 & $78(31.8)$ & $46(26.3)$ & 0.219 \\
\hline Vessel & $17(7.9)$ & I I (5.4) & 0.297 & $22(9)$ & $6(3.4)$ & 0.025 \\
\hline
\end{tabular}


Table 3 (Continued)

\begin{tabular}{|c|c|c|c|c|c|c|}
\hline \multirow[t]{2}{*}{ Characteristic } & \multicolumn{3}{|l|}{ NLR } & \multicolumn{3}{|l|}{ PLR } \\
\hline & $\leq 2.13(n=215)$ & $>2.13(n=205)$ & $P$-value & $\leq 140(n=245)$ & $>140(n=175)$ & $P$-value \\
\hline \multicolumn{7}{|c|}{ Tumor thrombus, n (\%) } \\
\hline Yes & $27(12.6)$ & $23(11.2)$ & 0.672 & $3 \mid(12.7)$ & $19(10.9)$ & 0.575 \\
\hline No & $188(87.4)$ & $182(88.8)$ & & $214(87.3)$ & $156(89.1)$ & \\
\hline \multicolumn{7}{|c|}{ CAI9-9 (U/mL), n (\%) } \\
\hline$\leq 37$ & $46(21.4)$ & $43(2 I)$ & 0.916 & $59(24.1)$ & $30(17.1)$ & 0.086 \\
\hline$>37$ & $169(78.6)$ & $162(79)$ & & $186(75.9)$ & $145(82.9)$ & \\
\hline \multicolumn{7}{|l|}{ CAI $25(\mathrm{U} / \mathrm{mL})$} \\
\hline$\leq 35$ & |7| (79.5) & $170(82.9)$ & 0.374 & $199(8 \mid .2)$ & $142(8 \mid .1)$ & 0.983 \\
\hline$>35$ & $44(20.5)$ & $35(17.1)$ & & $46(18.8)$ & $33(18.9)$ & \\
\hline \multicolumn{7}{|l|}{ CEA (ng/mL) } \\
\hline$\leq 5$ & $|4|$ (65.6) & 143 (69.8) & 0.361 & 161 (65.7) & $123(70.3)$ & 0.324 \\
\hline$>5$ & $74(34.4)$ & $62(30.2)$ & & $84(34.3)$ & $52(29.7)$ & \\
\hline
\end{tabular}

Note: Bold values $(P<0.05)$.

Abbreviations: CA 19-9, carbohydrate antigen 19-9; CAI25, carbohydrate antigen I25; CEA, carcinoembryonic antigen; ECOG PS, Eastern Cooperative Oncology Group performance status; NLR, neutrophil-lymphocyte ratio; PLR, platelet-lymphocyte ratio.

and chemokines; however, systemic levels of cytokines and chemokines are some way from routine clinical use. Therefore, several prognostic scores, based on shifts in the cellular components that occur during systemic inflammation have been proposed. ${ }^{19-21}$

Neutrophils, major components of the leukocyte population, can migrate from the venous system to tumors and may be triggered in the presence of cancer-related inflammatory factors, such as interleukin-6 (IL-6), granulocyte colonystimulating factor, and by myeloid growth factors produced during neoplastic processes. ${ }^{22}$ Neutrophils can release various factors, such as reactive oxygen species, leading to DNA damage, genetic instability, and tumor growth. ${ }^{23,24}$ In contrast, neutrophils interact with various other cell populations, leading to generation of numerous cytokines and effector molecules, particularly vascular endothelial growth factor (VEGF), which is important for tumor angiogenesis, growth, and metastasis. ${ }^{25}$ In addition, neutrophil subpopulations in the vicinity of the tumor microenvironment may suppress the antitumor immune responses of natural killer cells and activated $\mathrm{T}$ cells mediated by integrin Mac- 1 and hydrogen peroxide. ${ }^{26}$

Lymphocytes, which are the cellular mediators of immunosurveillance and immunoediting, have crucial roles in antitumor reactions through induction of cytotoxic cell death and inhibition of tumor cell proliferation and migration. ${ }^{8}$ During cancer-related inflammation, the presence of tumor-infiltrating lymphocytes with high $\mathrm{CD} 4+/ \mathrm{CD} 8+$ and Th2/Th1 ratios is indicative of poor prognosis. ${ }^{27}$

Platelets, the major transporter of VEGF, are regulated by pro-inflammatory cytokines, such as IL-1, IL-6, IL-11, and TNF- $\alpha .{ }^{28}$ Platelets can also contribute substantially to tumor vascular growth through secretion of a number of proangiogenic cytokines, including VEGF, platelet-derived growth factor, and thymidine phosphorylase..$^{29,30}$ Platelet aggregation also facilitates tumor cell adhesion to the vascular endothelium, which can act as a physiological shield, protecting tumor cells against NK cell-mediated lysis. ${ }^{31}$ Additionally, tumor cell infiltration and metastasis are induced via platelet-mediated activation of the $\mathrm{TGFb} / \mathrm{Smad}$ pathway. ${ }^{32}$

Overall, neutrophilia, thrombocytosis, and lymphopenia tend to contribute to cancer-related inflammatory responses, and the NLR and PLR, simple derivatives of routine blood counts, can reflect the dynamic balance between anti- and pro-tumor functions of the immune system. ${ }^{33}$

Currently, there is no consensus on the appropriate cutoff values for NLR and PLR for prediction of prognosis in patients with PDAC; thus, various thresholds have been used in previous studies. For NLR, many studies have used a cutoff value of 5.0, ${ }^{34-36}$ while others used values varying from 2.2 to 4.0. ${ }^{15,37-39}$ PLR cutoff values of 150,160 , and 300 have been used in previous studies. ${ }^{14,15,36,40,41}$ In some studies, patients were even categorized according to quartiles or mean values of NLR or PLR; ${ }^{42}$ however, despite these differences in cutoff values, all studies reported worse survival when NLR or PLR exceeded the threshold. Unlike studies that set cutoff values for NLR and PLR empirically, we used time-dependent ROC to determine the values, and calculated optimal thresholds of 2.13 for NLR and 140 for PLR. The AUCs of NLR and PLR were 0.743 and 0.715 , respectively, indicating that both indices are promising predictors of DFS in patients with resectable PDAC. In contrast, the AUCs of LMR and 
A
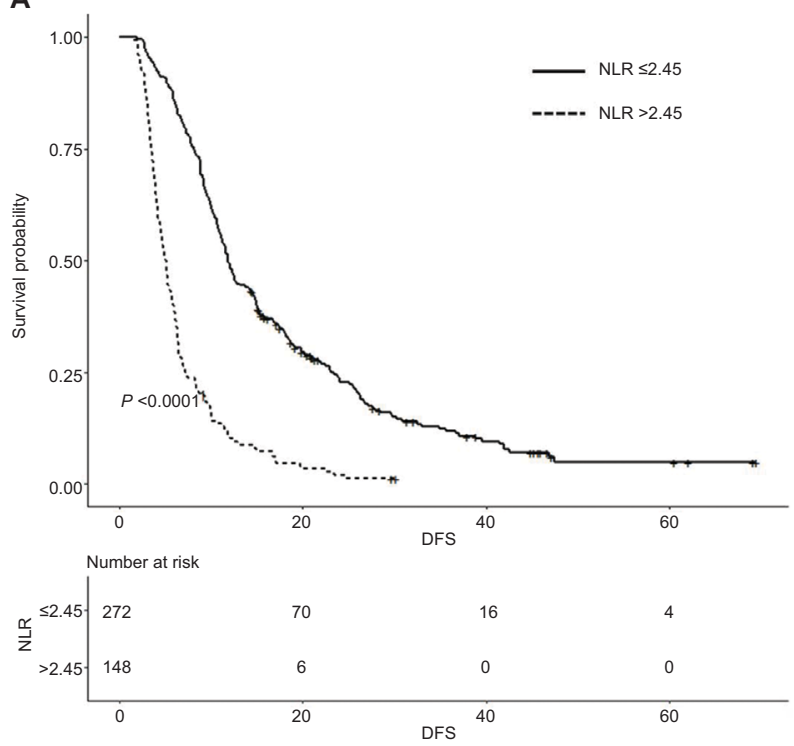

C
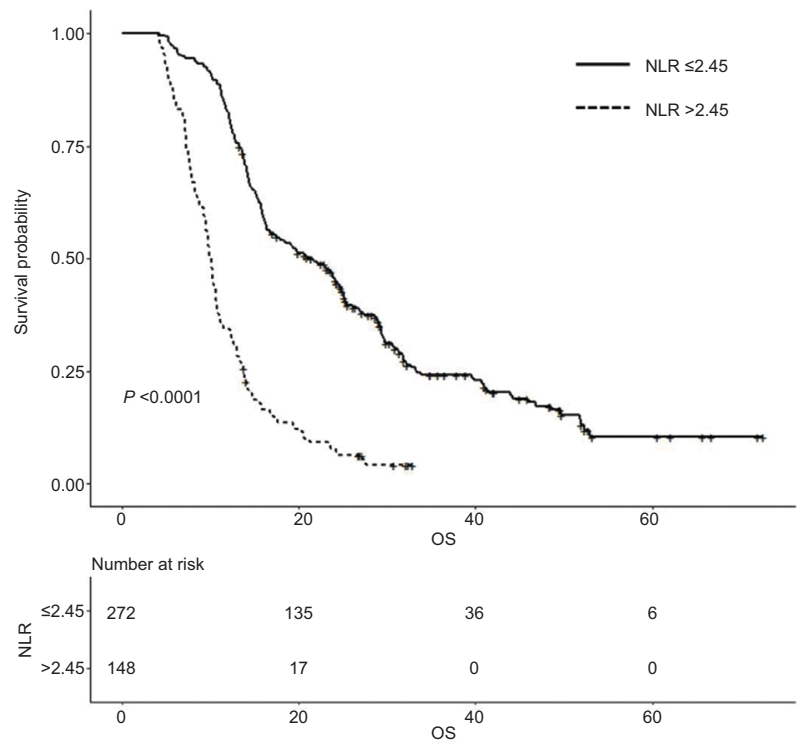

B
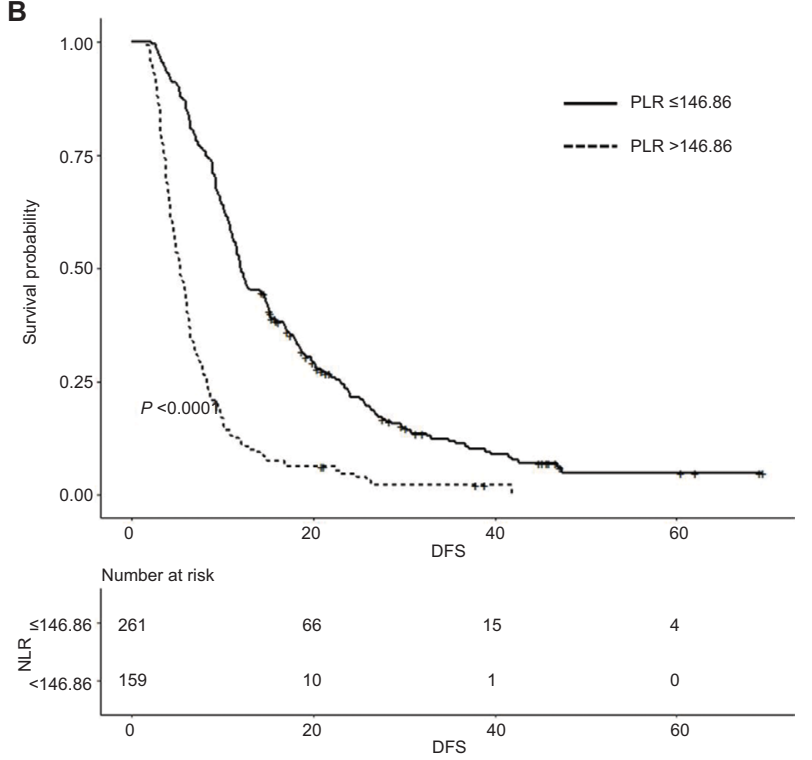

D
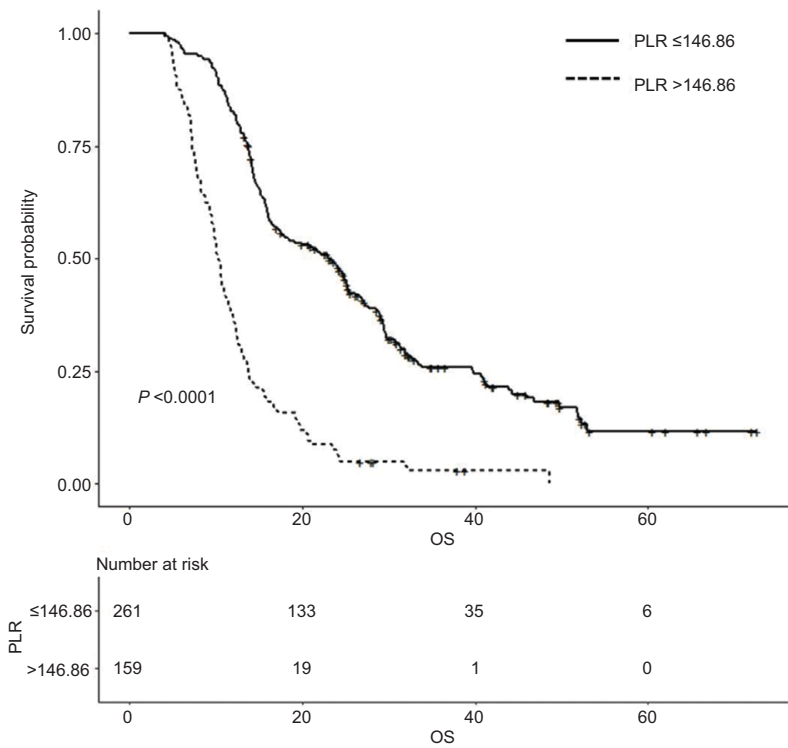

Figure 2 Kaplan-Meier curves of NLR and PLR for DFS and OS.

Notes: (A) NLR for DFS, (B) PLR for DFS, (C) NLR for OS, (D) PLR for OS

Abbreviations: DFS, disease-free survival; NLR, neutrophil-lymphocyte ratio; OS, overall survival; PLR, platelet-lymphocyte ratio.

PNI were both $<0.5$, demonstrating no prognostic power for predicting DFS. Further survival analyses showed that high NLR and high PLR (above the threshold values) were significantly associated with impaired DFS and OS, independent of a wide range of potential confounding factors. These results are consistent with previous publications. ${ }^{15,37}$ Interestingly, in analysis stratified by TNM stage, we demonstrated that patient subgroups with stage I-II tumors presented different DFS and OS according to their NLR or PLR values; however, these associations were not observed for patients with stage III tumors. The small number of patients with stage III disease may be an important factor contributing to this phenomenon. Although these results are insufficient to directly influence clinical practice at present, they provide valuable information regarding clinical risk stratification to facilitate improved personalized medical care, such as a closer follow-up once high NLR and/or high PLR are identified, even among patients with the same stage tumor. 
Table 4 Multivariate analyses for DFS and OS

\begin{tabular}{|c|c|c|c|c|c|c|c|c|}
\hline \multirow[t]{3}{*}{ Characteristic } & \multicolumn{4}{|l|}{ DFS } & \multicolumn{4}{|l|}{ OS } \\
\hline & \multicolumn{2}{|l|}{ Univariate } & \multicolumn{2}{|l|}{ Multivariate } & \multicolumn{2}{|l|}{ Univariate } & \multicolumn{2}{|l|}{ Multivariate } \\
\hline & HR (95\% Cl) & $P$-value & HR (95\% Cl) & $P$-value & HR (95\% Cl) & $P$-value & HR (95\% Cl) & $P$-value \\
\hline \multicolumn{9}{|l|}{ Age (years), n (\%) } \\
\hline$\leq 50$ & I (ref) & & \multirow[t]{3}{*}{ - } & & I (ref) & & \multirow[t]{3}{*}{ - } & \\
\hline $5 \mathrm{I}-70$ & I.II (0.83-I.47) & 0.492 & & & I.12(0.83-I.5I) & 0.45 & & \\
\hline$>70$ & $0.97(0.65-1.44)$ & 0.879 & & & $0.91(0.60-1.38)$ & 0.643 & & \\
\hline \multicolumn{9}{|l|}{ Sex, n (\%) } \\
\hline Female & I (ref) & 0.193 & \multirow[t]{2}{*}{ - } & & I (ref) & 0.64 & \multirow[t]{2}{*}{ - } & \\
\hline Male & $0.87(0.7 \mathrm{I}-\mathrm{I} .07)$ & & & & $1.05(0.85-1.32)$ & & & \\
\hline \multicolumn{9}{|l|}{ Smoking, n (\%) } \\
\hline Yes & I (ref) & 0.175 & \multirow[t]{2}{*}{ - } & & I (ref) & 0.061 & \multirow[t]{2}{*}{ - } & \\
\hline No & $1.20(0.92-1.56)$ & & & & $1.30(0.99-1.70)$ & & & \\
\hline Alcohol consumption & & & & & & & & \\
\hline n (\%) & & & & & & & & \\
\hline Yes & I (ref) & 0.557 & - & & I (ref) & 0.512 & - & \\
\hline No & $0.94(0.75-1.17)$ & & & & $0.93(0.73-1.17)$ & & & \\
\hline ECOG PS, n (\%) & & & & & & & & \\
\hline $0-1$ & I (ref) & $<0.001$ & I (ref) & $<0.001$ & I (ref) & $<0.001$ & I (ref) & $<0.001$ \\
\hline$\geq 2$ & $2.84(2.15-3.76)$ & & $2.44(1.81-3.29)$ & & $2.61(1.96-3.47)$ & & $2.09(1.54-2.84)$ & \\
\hline Tumor location, n (\% & & & & & & & & \\
\hline Head & I (ref) & $0.56 \mathrm{I}$ & _- & & I (ref) & 0.216 & _ & \\
\hline Body and/or tail & $1.07(0.86-1.32)$ & & & & $0.86(0.68-1.09)$ & & & \\
\hline Maximum diameter & (\%) & & & & & & & \\
\hline$<2$ & I (ref) & & - & & I (ref) & & - & \\
\hline $2-4$ & $1.29(0.85-1.96)$ & 0.2349 & & & $1.05(0.70-1.55)$ & 0.825 & & \\
\hline$>4$ & $1.36(0.87-2.11)$ & 0.173 & & & $1.19(0.79-1.81)$ & 0.403 & & \\
\hline Differentiation, $\mathrm{n}(\%)$ & & & & & & & & \\
\hline Well & I (ref) & 0.04 & I (ref) & 0.003 & I (ref) & 0.116 & I (ref) & 0.027 \\
\hline Poor & $1.23(1.01-1.5 \mathrm{I})$ & & $1.36(1.11-1.67)$ & & $1.19(0.96-1.46)$ & & $1.28(1.03-1.58)$ & \\
\hline T stage, n (\%) & & & & & & & & \\
\hline TI & I (ref) & & _ & & I (ref) & & _- & \\
\hline $\mathrm{T} 2$ & $1.21(0.85-1.72)$ & 0.288 & & & I.0I (0.72-I.42) & 0.958 & & \\
\hline T3 & $1.32(0.91-1.92)$ & 0.139 & & & $1.21(0.85-1.74)$ & 0.292 & & \\
\hline $\mathrm{T} 4$ & $18.22(8.77-37.83)$ & $<0.001$ & & & $12.72(6.24--25.93)$ & $<0.001$ & & \\
\hline $\mathrm{N}$ stage $\mathrm{n}(\%)$ & & & & & & & & \\
\hline No & I (ref) & & - & & I (ref) & & _ & \\
\hline $\mathrm{NI}$ & $1.10(0.86-1.39)$ & 0.45 & & & I.23 (0.97-I.58) & 0.092 & & \\
\hline $\mathrm{N} 2$ & $4.05(2.80-5.85)$ & $<0.001$ & & & $3.27(2.25-4.75)$ & $<0.001$ & & \\
\hline pTNM stage, n (\%) & & & & & & & & \\
\hline I & I (ref) & & I (ref) & & I (ref) & & I (ref) & \\
\hline ॥ & $1.29(1.04-1.60)$ & 0.02 & $1.42(1.14-1.77)$ & 0.002 & $1.42(1.13-1.78)$ & 0.002 & $1.47(1.17-1.85)$ & 0.001 \\
\hline III & $5.71(4.02-8.09)$ & $<0.001$ & $4.69(3.26-6.76)$ & $<0.001$ & $4.72(3.3 I-6.75)$ & $<0.001$ & $3.25(2.25-4.68)$ & $<0.001$ \\
\hline Tumor extension, $\mathrm{n}$ & & & & & & & & \\
\hline Choledochal duct & $0.90(0.73-1.1 \mathrm{I})$ & 0.334 & _ & & $0.90(0.72-1.13)$ & 0.364 & _ & \\
\hline Duodenum & I. $15(0.93-1.42)$ & 0.194 & - & & $1.04(0.83-1.30)$ & 0.744 & - & \\
\hline Peripheral tissue & $1.16(0.98-1.24)$ & 0.058 & - & & $1.33(1.07-1.66)$ & 0.009 & $1.25(0.99-1.56)$ & 0.054 \\
\hline Nerve & $1.09(0.87-1.36)$ & 0.446 & - & & $1.07(0.85-1.35)$ & 0.585 & - & \\
\hline Vessel & $0.73(0.48-1.10)$ & 0.136 & - & & $0.82(0.53-1.26)$ & 0.356 & - & \\
\hline Tumor thrombus, $\mathrm{n}$ & & & & & & & & \\
\hline Yes & I (ref) & 0.151 & - & & I (ref) & 0.137 & _- & \\
\hline No & $0.79(0.57-1.09)$ & & & & $0.77(0.54-1.09)$ & & & \\
\hline CAI9-9 (U/mL). n (\% & & & & & & & & \\
\hline$\leq 37$ & I (ref) & 0.768 & - & & I (ref) & 0.502 & _ & \\
\hline$>37$ & $\mathrm{I} .04(0.8 \mathrm{I}-\mathrm{I} .32)$ & & & & $\mathrm{I} .09(0.85-1.4 \mathrm{I})$ & & & \\
\hline CAI $25(\mathrm{U} / \mathrm{mL})$ & & & & & & & & \\
\hline$\leq 35$ & I (ref) & 0.362 & _ & & I (ref) & 0.134 & _ & \\
\hline$>35$ & I.13 (0.87-I.45) & & & & $1.22(0.94-1.58)$ & & & \\
\hline
\end{tabular}


Table 4 (Continued)

\begin{tabular}{|c|c|c|c|c|c|c|c|c|}
\hline \multirow[t]{3}{*}{ Characteristic } & \multicolumn{4}{|l|}{ DFS } & \multicolumn{4}{|l|}{ os } \\
\hline & \multicolumn{2}{|l|}{ Univariate } & \multicolumn{2}{|l|}{ Multivariate } & \multicolumn{2}{|l|}{ Univariate } & \multicolumn{2}{|l|}{ Multivariate } \\
\hline & HR (95\% CI) & $P$-value & HR (95\% Cl) & $P$-value & HR (95\% CI) & $P$-value & HR (95\% CI) & $P$-value \\
\hline \multicolumn{9}{|l|}{ CEA (ng/mL) } \\
\hline$\leq 5$ & I (ref) & 0.503 & - & & I (ref) & 0.439 & - & \\
\hline$>5$ & $1.08(0.87-1.33)$ & & & & $1.09(0.87-1.37)$ & & & \\
\hline \multicolumn{9}{|c|}{ Adjuvant treatment } \\
\hline No & I (ref) & $<0.001$ & I (ref) & $<0.001$ & I (ref) & $<0.001$ & I (ref) & $<0.001$ \\
\hline Yes & $0.63(0.50-0.79)$ & & $0.6(0.48-0.76)$ & & $0.62(0.49-0.79)$ & & $0.61(0.47-0.78)$ & \\
\hline \multicolumn{9}{|l|}{ NLR } \\
\hline$\leq 2.13$ & I (ref) & $<0.001$ & I (ref) & $<0.001$ & I (ref) & $<0.001$ & I (ref) & $<0.001$ \\
\hline$>2.13$ & $2.43(1.98-2.99)$ & & $1.59(1.27-2.00)$ & & $2.65(2.14-3.29)$ & & $1.70(1.33-2.16)$ & \\
\hline \multicolumn{9}{|l|}{ PLR } \\
\hline$\leq 140$ & I (ref) & $<0.001$ & I (ref) & $<0.001$ & I (ref) & $<0.001$ & I (ref) & $<0.001$ \\
\hline$>140$ & $2.54(2.06-3.13)$ & & $1.93(1.53-2.45)$ & & $2.93(2.35-3.64)$ & & $2.23(1.74-2.85)$ & \\
\hline
\end{tabular}

Note: Bold values $(P<0.05)$.

Abbreviations: CA19-9, carbohydrate antigen 19-9; CAI25, carbohydrate antigen I25; CEA, carcinoembryonic antigen; CI, confidence interval; DFS, disease-free survival; ECOG PS, Eastern Cooperative Oncology Group performance status; HR, hazard ratio; NLR, neutrophil-lymphocyte ratio; OS, overall survival; PLR, platelet-lymphocyte ratio.

Table 5 Multivariate analyses of NLR and PLR for DFS and OS stratified by PTNM stage

\begin{tabular}{|c|c|c|c|c|c|c|c|}
\hline \multirow[t]{3}{*}{ Stage } & \multirow[t]{3}{*}{ Survival } & \multicolumn{2}{|l|}{ NLR } & \multirow[t]{3}{*}{$P$-value } & \multicolumn{2}{|l|}{ PLR } & \multirow[t]{3}{*}{$P$-value } \\
\hline & & $\leq 2.13$ & $>2.13$ & & $\leq 140$ & $>140$ & \\
\hline & & & HR (95\% Cl) & & & HR (95\% Cl) & \\
\hline \multirow[t]{2}{*}{1} & DFS & I (ref) & $2.57(1.83-3.60)$ & $<0.001$ & I (ref) & $3.82(2.69-5.4 I)$ & $<0.001$ \\
\hline & OS & I (ref) & $2.80(1.95-4.00)$ & $<0.001$ & I (ref) & $5.06(3.40-7.53)$ & $<0.001$ \\
\hline \multirow[t]{2}{*}{ II } & DFS & I (ref) & $2.0 \mathrm{I}(\mathrm{I} .44-2.8 \mathrm{I})$ & $<0.001$ & I (ref) & $2.06(1.4 \mathrm{I}-3.00)$ & $<0.001$ \\
\hline & OS & I (ref) & $2.06(1.46-2.92)$ & $<0.001$ & I (ref) & $2.24(1.54-3.28)$ & $<0.001$ \\
\hline \multirow[t]{2}{*}{ III } & DFS & I (ref) & $0.80(0.34-1.88)$ & 0.609 & I (ref) & $1.46(0.67-3.18)$ & 0.344 \\
\hline & OS & I (ref) & $1.60(0.65-3.95)$ & 0.305 & I (ref) & $2.11(0.98-4.55)$ & 0.058 \\
\hline
\end{tabular}

Note: Model adjusted for age, sex, ECOG PS score, smoking status, alcohol consumption, differentiation, and adjuvant treatment.

Abbreviations: Cl, confidence interval; DFS, disease-free survival; ECOG PS, Eastern Cooperative Oncology Group performance status; HR, hazard ratio; NLR, neutrophillymphocyte ratio; OS, overall survival; PLR, platelet-lymphocyte ratio.

Accurate information on prognostication is useful for medical decision-making and clinical counseling. Nomograms, statistical instruments that can evaluate multiple relevant clinical factors and predict the continuous probability of a particular outcome for an individual patient, have been used in an extensive array of applications, including cancer. ${ }^{43}$ To allow physicians to tailor prognostication for patients with resectable PDAC who maintain an underlying constant systemic inflammatory status, we established a nomogram incorporating NLR and PLR and compared its discriminatory ability to an instrument not incorporating NLR and PLR. In our study, the independent predictors, ECOG PS score, tumor stage, differentiation, adjuvant treatment, NLR, and PLR, were incorporated into the two nomograms. The AUCs of the nomograms including or excluding NLR and PLR were 0.804 and 0.711 , respectively, indicating that the former had superior discrimination ability for prediction of clinical outcomes. In addition, the nomogram incorporating NLR and PLR performed well on bootstrap validation, with a bootstrap-corrected AUC of 0.803 . Thus, for example, a patient with an ECOG PS score of $0-1$ ( 0 points), stage II tumor (65 points), well-differentiated tumor ( 0 points), NLR $>2.13$ (90 points), PLR $\leq 140$ (0 points), and no adjuvant treatment ( 95 points) would score 250 total points, which converts to a probability for 1 -year DFS of $<10 \%$. The predictors included in our nomogram are all available in routine clinical practice; therefore, we believe that it can be easily used by clinicians to make accurate individualized estimates of prognosis.

The strengths of our study are the large sample size and the long follow-up period. Moreover, our prediction model enables surgical PDAC patients to be monitored easily on an individual basis. Nevertheless, the investigation had several limitations that merit emphasis. First, although the 
A

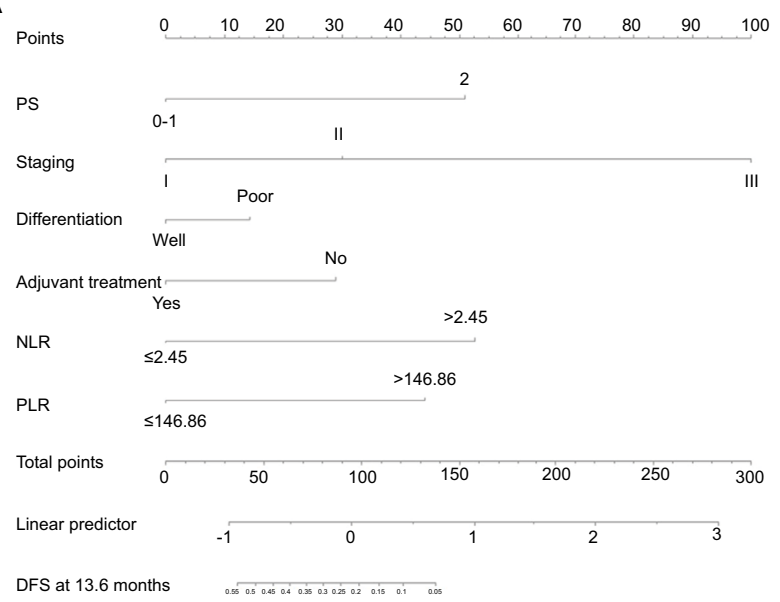

C

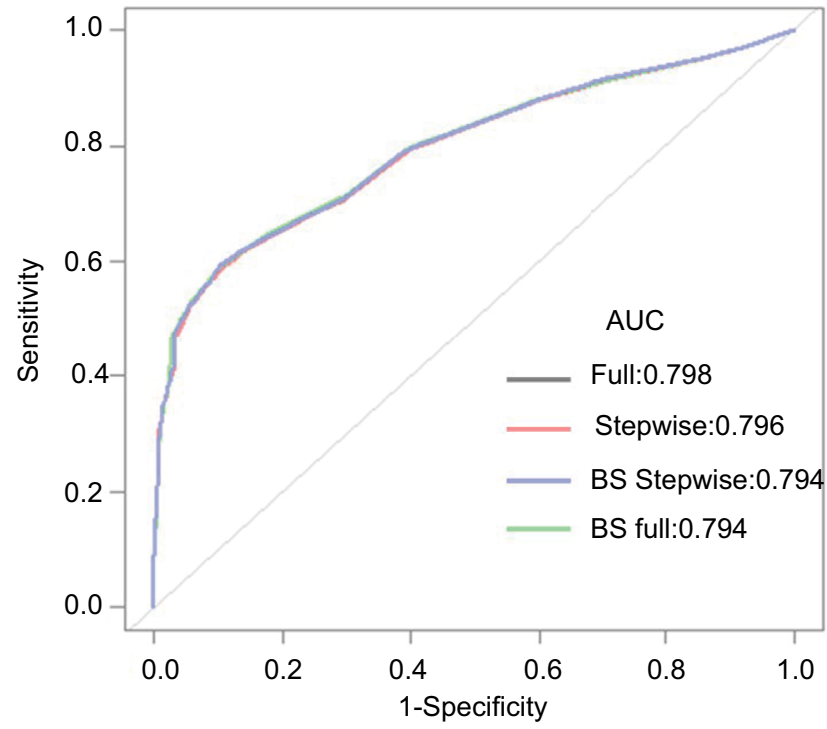

B

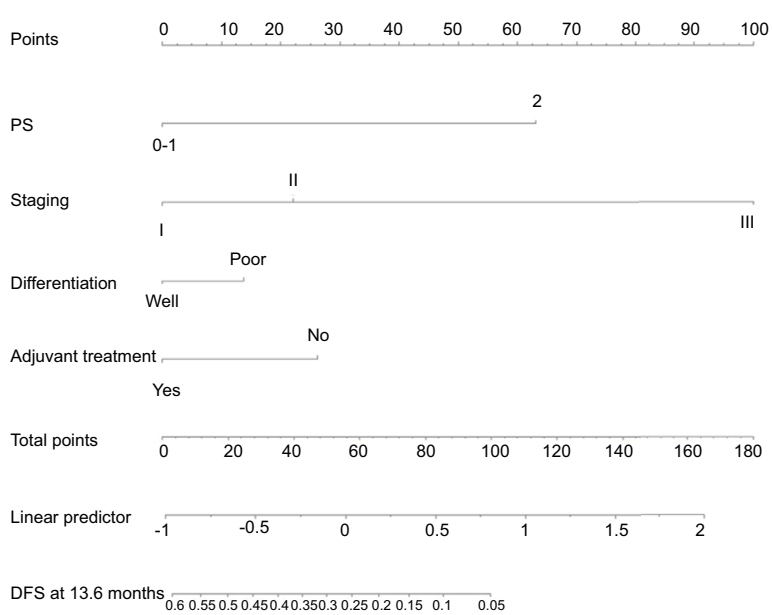

D

Model 2

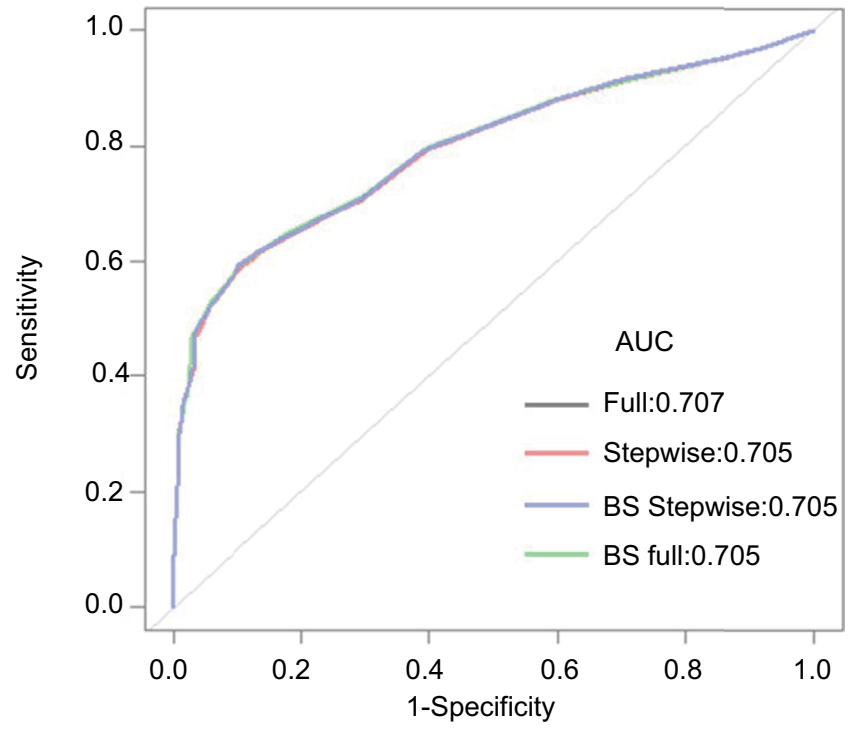

Figure 3 Nomograms and their accuracy for predicting I-year DFS in patients with resectable PDAC.

Notes: (A) Nomogram including NLR and PLR, (B) nomogram without NLR and PLR, (C) bootstrap-corrected AUCs for model including NLR and PLR, (D) bootstrapcorrected (BS) AUCs for model without NLR and PLR.

Abbreviations: AUCs, areas under the curve; DFS, disease-free survival; ECOG PS, Eastern Cooperative Oncology Group performance status; NLR, neutrophil-lymphocyte ratio; PDAC, pancreatic ductal adenocarcinoma; PLR, platelet-lymphocyte ratio.

current study adjusted for a considerable number of potential confounders, we cannot rule out the possibility that the results may have been affected by other variables that were not included in the analyses. Second, patients were included in the database over a period of 10 years; therefore, it is possible that advances in surgical techniques and medical oncology have combined to improve survival of patients with resected PDAC. Hence, we used the bootstrapping technique to obtain an almost unbiased internal assessment of accuracy. Although the results of the bootstrapping correction procedure are promising, they do not negate the need for future studies to externally validate whether our nomograms can be generalized to new patient populations. Furthermore, our conclusions were solely drawn from the objective clinical data, as it was beyond the scope of our study to elucidate the potential mechanism underlying the prognostic value of inflammatory markers.

\section{Conclusion}

In the present study, we demonstrated that high NLR and high PLR are clinically important predictors of poor survival in patients with resectable PDAC. Additionally, our 
predictive nomogram including NLR and PLR can objectively and reliably predict DFS among PDAC patients after curative surgery, and performed well on internal validation. Therefore, with their advantages of cost-effectiveness and simplicity, NLR and PLR may be useful for stratification of high-risk subpopulations of patients with resectable PDAC in terms of recurrence and poor survival, even where tumors are the same stage. A further large-scale prospective validation study is needed to determine whether these results are widely applicable.

\section{Author contributions}

Both authors contributed toward data analysis, drafting and revising the paper and agree to be accountable for all aspects of the work.

\section{Disclosure}

The authors report no conflicts of interest in this work.

\section{References}

1. American Cancer Society [homepage on the Internet]. Cancer Statistics Center. Available from: http://cancerstatisticscenter.cancer.org. Accessed August 7, 2018.

2. Sharma C, Eltawil KM, Renfrew PD, Walsh MJ, Molinari M. Advances in diagnosis, treatment and palliation of pancreatic carcinoma: 1990-2010. World J Gastroenterol. 2011;17(7):867-897.

3. Katz MH, Wang H, Fleming JB, et al. Long-term survival after multidisciplinary management of resected pancreatic adenocarcinoma. Ann Surg Oncol. 2009;16(4):836-847.

4. Fortner JG, Klimstra DS, Senie RT, Maclean BJ. Tumor size is the primary prognosticator for pancreatic cancer after regional pancreatectomy. Ann Surg. 1996;223(2):147-153.

5. Raut CP, Tseng JF, Sun CC, et al. Impact of resection status on pattern of failure and survival after pancreaticoduodenectomy for pancreatic adenocarcinoma. Ann Surg. 2007;246(1):52-60.

6. Schmidt CM, Matos JM, Bentrem DJ, Talamonti MS, Lillemoe KD, Bilimoria KY. Acinar cell carcinoma of the pancreas in the United States: prognostic factors and comparison to ductal adenocarcinoma. J Gastrointest Surg. 2008;12(12):2078-2086.

7. Mckay CJ, Glen P, McMillan DC. Chronic inflammation and pancreatic cancer. Best Pract Res Clin Gastroenterol. 2008;22(1):65-73.

8. Mantovani A, Allavena P, Sica A, Balkwill F. Cancer-related inflammation. Nature. 2008;454(7203):436-444.

9. Grivennikov SI, Greten FR, Karin M, Immunity KM. Immunity, inflammation, and cancer. Cell. 2010;140(6):883-899.

10. Proctor MJ, Morrison DS, Talwar D, et al. A comparison of inflammation-based prognostic scores in patients with cancer. A Glasgow Inflammation Outcome Study. Eur J Cancer. 2011;47(17):2633-2641.

11. McMillan DC. Systemic inflammation, nutritional status and survival in patients with cancer. Curr Opin Clin Nutr Metab Care. 2009;12(3): 223-226.

12. Proctor MJ, Morrison DS, Talwar D, et al. An inflammation-based prognostic score (mGPS) predicts cancer survival independent of tumour site: a Glasgow Inflammation Outcome Study. $\mathrm{Br} J$ Cancer. 2011;104(4):726-734.

13. Kasymjanova G, MacDonald N, Agulnik JS, et al. The predictive value of pre-treatment inflammatory markers in advanced non-small-cell lung cancer. Curr Oncol. 2010;17(4):52-58.
14. Stotz M, Gerger A, Eisner F, et al. Increased neutrophil-lymphocyte ratio is a poor prognostic factor in patients with primary operable and inoperable pancreatic cancer. Br J Cancer. 2013;109(2):416-421.

15. Smith RA, Bosonnet L, Raraty M, et al. Preoperative plateletlymphocyte ratio is an independent significant prognostic marker in resected pancreatic ductal adenocarcinoma. Am J Surg. 2009;197(4): 466-472.

16. Elinav E, Nowarski R, Thaiss CA, Hu B, Jin C, Flavell RA. Inflammation-induced cancer: crosstalk between tumours, immune cells and microorganisms. Nat Rev Cancer. 2013;13(11):759-771.

17. Gencer D, Kästle-Larralde N, Pilz LR, et al. Presentation, treatment, and analysis of prognostic factors of terminally ill patients with gastrointestinal tumors. Onkologie. 2009;32(7):380-386.

18. Siddiqui A, Heinzerling J, Livingston EH, Huerta S. Predictors of early mortality in veteran patients with pancreatic cancer. Am J Surg. 2007;194(3):362-366.

19. Leitch EF, Chakrabarti M, Crozier JE, et al. Comparison of the prognostic value of selected markers of the systemic inflammatory response in patients with colorectal cancer. Br J Cancer. 2007;97(9): $1266-1270$.

20. Condeelis J, Pollard JW. Macrophages: obligate partners for tumor cell migration, invasion, and metastasis. Cell. 2006;124(2):263-266.

21. Huh JW, Lee JH, Kim HR. Prognostic significance of tumor-infiltrating lymphocytes for patients with colorectal cancer. Arch Surg. 2012;147(4):366-372.

22. Liang W, Ferrara N. The Complex Role of Neutrophils in Tumor Angiogenesis and Metastasis. Cancer Immunol Res. 2016;4(2):83-91.

23. McColl SR, Paquin R, Ménard C, Beaulieu AD. Human neutrophils produce high levels of the interleukin 1 receptor antagonist in response to granulocyte/macrophage colony-stimulating factor and tumor necrosis factor alpha. J Exp Med. 1992;176(2):593-598.

24. Weitzman SA, Gordon LI. Inflammation and cancer: role of phagocyte-generated oxidants in carcinogenesis. Blood. 1990;76(4): 655-663.

25. Kusumanto YH, Dam WA, Hospers GA, Meijer C, Mulder NH. Platelets and granulocytes, in particular the neutrophils, form important compartments for circulating vascular endothelial growth factor. Angiogenesis. 2003;6(4):283-287.

26. Pillay J, Kamp VM, van Hoffen E, et al. A subset of neutrophils in human systemic inflammation inhibits T cell responses through Mac-1. J Clin Invest. 2012;122(1):327-336.

27. Kohrt HE, Nouri N, Nowels K, Johnson D, Holmes S, Lee PP. Profile of immune cells in axillary lymph nodes predicts disease-free survival in breast cancer. PLoS Med. 2005;2(9):e284.

28. Bambace NM, Holmes CE. The platelet contribution to cancer progression. J Thromb Haemost. 2011;9(2):237-249.

29. Möhle R, Green D, Moore MA, Nachman RL, Rafii S. Constitutive production and thrombin-induced release of vascular endothelial growth factor by human megakaryocytes and platelets. Proc Natl Acad Sci U $S A$. 1997;94(2):663-668.

30. Okamoto E, Osaki M, Kase S, Adachi H, Kaibara N, Ito H. Thymidine phosphorylase expression causes both the increase of intratumoral microvessels and decrease of apoptosis in human esophageal carcinomas. Pathol Int. 2001;51(3):158-164.

31. Gay LJ, Felding-Habermann B. Contribution of platelets to tumour metastasis. Nat Rev Cancer. 2011;11(2):123-134.

32. Labelle M, Begum S, Hynes RO. Direct signaling between platelets and cancer cells induces an epithelial-mesenchymal-like transition and promotes metastasis. Cancer Cell. 2011;20(5):576-590.

33. Hanahan D, Coussens LM. Accessories to the crime: functions of cells recruited to the tumor microenvironment. Cancer Cell. 2012;21(3):309-322.

34. Garcea G, Ladwa N, Neal CP, Metcalfe MS, Dennison AR, Berry DP. Preoperative neutrophil-to-lymphocyte ratio (NLR) is associated with reduced disease-free survival following curative resection of pancreatic adenocarcinoma. World J Surg. 2011;35(4):868-872. 
35. Clark EJ, Connor S, Taylor MA, Madhavan KK, Garden OJ, Parks RW. Preoperative lymphocyte count as a prognostic factor in resected pancreatic ductal adenocarcinoma. HPB. 2007;9(6):456-460.

36. Jamieson NB, Denley SM, Logue J, et al. A prospective comparison of the prognostic value of tumor- and patient-related factors in patients undergoing potentially curative surgery for pancreatic ductal adenocarcinoma. Ann Surg Oncol. 2011;18(8):2318-2328.

37. Szkandera J, Stotz M, Eisner F, et al. External validation of the derived neutrophil to lymphocyte ratio as a prognostic marker on a large cohort of pancreatic cancer patients. PLoS One. 2013;8(11):e78225.

38. Teo M, Mohd Sharial MS, McDonnell F, Conlon KC, Ridgway PF, McDermott RS. Prognostic role of neutrophil-to-lymphocyte ratio in advanced pancreatic ductal adenocarcinoma: impact of baseline fluctuation and changes during chemotherapy. Tumori. 2013;99(4): $516-522$.
39. Bhatti I, Peacock O, Lloyd G, Larvin M, Hall RI. Preoperative hematologic markers as independent predictors of prognosis in resected pancreatic ductal adenocarcinoma: neutrophil-lymphocyte versus platelet-lymphocyte ratio. Am J Surg. 2010;200(2):197-203.

40. Wang DS, Luo HY, Qiu MZ, et al. Comparison of the prognostic values of various inflammation based factors in patients with pancreatic cancer. Med Oncol. 2012;29(5):3092-3100.

41. Aliustaoglu M, Bilici A, Seker M, et al. The association of pre-treatment peripheral blood markers with survival in patients with pancreatic cancer. Hepatogastroenterology. 2010;57(99-100):640-645.

42. Asaoka T, Miyamoto A, Maeda S, et al. Prognostic impact of preoperative NLR and CA19-9 in pancreatic cancer. Pancreatology. 2016;16(3):434-440.

43. Iasonos A, Schrag D, Raj GV, Panageas KS. How to build and interpret a nomogram for cancer prognosis. J Clin Oncol. 2008;26(8):1364-1370.
Cancer Management and Research

\section{Publish your work in this journal}

Cancer Management and Research is an international, peer-reviewed open access journal focusing on cancer research and the optimal use of preventative and integrated treatment interventions to achieve improved outcomes, enhanced survival and quality of life for the cancer patient The manuscript management system is completely online and includes

\section{Dovepress}

a very quick and fair peer-review system, which is all easy to use. Visit http://www.dovepress.com/testimonials.php to read real quotes from published authors. 